\title{
An Underestimated Tool: Body Language in Classroom during Teaching and Learning
}

\author{
Mustafa Altun ${ }^{1}$ \\ ${ }^{1}$ Department of English Language Teaching, Ishik University, Erbil, Iraq \\ Correspondence: Mustafa Altun, Ishik University, Erbil, Iraq. \\ Email: mustafa.altun@ishik.edu.iq
}

Received: June 9, $2019 \quad$ Accepted: August 20, $2019 \quad$ Online Published: September 1, 2019

doi: 10.23918/ijsses.v6i1p155

\begin{abstract}
For many people who work as presenters, lecturers, or public speakers, they use many tools to deliver their messages besides verbal language. Body Language is the nonverbal aspect of any face-to-face communication, and it is an effective way to establish an authentic relationship between the addresser and the addressee. We learn body language from our childhood just like learning spoken language, without realizing that we do. The difference is that while our linguistic mistakes are corrected, our body language mistakes can be missed or misinterpreted, so we grow up communicating not in the way we should. This paper will tackle types of body language and deal with some differences that are encountered in different cultures in terms of using body movements that accompany speech. The paper will be devoted to exploring how to read basic body language, and as an example, reading body language in terms of gender differences will be discussed. It will also provide information about the teacher's nonverbal behavior, and finally will deal briefly with the student's body language and how it is recognized by the teacher.
\end{abstract}

Keywords: Drama, Classroom Management, Body Language, Verbal Nonverbal Communication, Multiple Intelligence.

\section{Introduction}

Communication between human beings is mostly coordinated with their behaviors. Body movement in face-to-face communication is essential items in conveying information. The informative communication is important and it is widely transferred through verbal language. Emotional and attitudinal communications are expressed primarily by other means refer to as Paralanguage and Kinesics (Key, 1970, p. 16). Paralanguage, as defined by the Oxford Dictionary is the non-lexical component of communication by speech. Paralanguage is mostly concerned with voice nuances as a means of expressing feelings and thoughts. Whereas Kinesics, which is the anthropological term for Body Language, appeared with the development of paralinguistic studies in 1952 by the American Anthropologist Ray Birdwhistell; it refers to the study of gestures and facial expressions and other body movements. Body language is an old aged science; it was first to appear as Physiognomy, which is the art of discovering temperament and character from the outward appearance (Merriam-Webster, n.d). This science originated from ancient Greeks. It was then revived at the beginning of the 19th century by the Swiss pastor and poet Johann Kaspar Lavater in his book Physiognomische Fragmente zur Beförderung der Menschenkenntnis und Menschenliebe 17751778 (Chisholm, 1911). The science of Physiognomy was related to other science called Phrenology, literally "mental science" originated by Gall and Spurzheim. It was based on some brain measurements 
and of its development as an organ (Dictionary.com, n.d). This science of Phrenology was considered a pseudoscience because it did not rely on a valid scientific method. Since Physiognomy was associated with Phrenology it was written-off for a period of time. The science of Physiognomy was revived by many researchers around the world as a way to know human beings' behaviors. It is called the New Physiognomy and it has a tied relation to the movement of the human body in communication (Wiseman, 2009) which is the core of the study of modern body language. By the end of the twentieth century, researchers like Albert Mehrabian and Birdwhistell have taken Darwin's observations from his book The Expression of the Emotions in Man and Animals (1872), on nonverbal cues and signals. Birdwhistell found out that the amount of non-verbal communication is bigger than the verbal one. He estimated that the normal person speaks an average of 10 or 11 minutes a day, and the average sentence takes about 2.5 seconds. Mehrabian have found, also through many experiments, that words are only $7 \%$ of the whole communication, the rest 35 and 65 are divided between vocal variety and body movements (gestures, postures, facial expressions...) (Wainwright, 2010). Furthermore, it has been found that some of our inborn gestures and facial expressions are similar to those of primates (apes, chimpanzees...) as referred by Charles Darwin in his book The Expressions of the Emotions in Man and Animals. Mammalians show some facial expressions and even significant body movements. Aggression signs, for instance, are characterized by the opening of the mouth, direct staring, and forward erection of ears (Waller, 2013). Humans are just the evolutional version primates according to Darwin's evolutionary theory. In Kinesics, a "kine" is the smallest observable unit. Terms such as phoneme in Phonology and morpheme in morphology, there is also the term "kineme" in Kinesics. A kineme is a movement associated with meaning, and it is considered to be a basic unit in studying different criteria of body movement. A person is kinetically-mature means that he/she knows how to use her/his body and knows when and where a movement is done.

\section{Literature Review}

\subsection{Types of Body Language}

What types of body language can be recognized and deciphered? Knowing about the types and each unit in reading body language helps us control our nonverbal behavior through our communication.

\subsubsection{Postures, Gestures and Head Tilt}

\subsubsection{Postures}

Each person has its repertoire of postures, and they are considered as a tool to characterize and recognize ourselves. It also helps us give good impressions about us in our social lives. Some people have what Birdswhistell described "Preferred Postures", those people are said to be reflected by their past. For example, those who faced bullying, or were forced to stand up for themselves as children, may show curved, defensive postures and will imply on everything they do in their daily lives.

There are three kinds of postures:

Standing postures, sitting postures, and lying down postures. This section will mainly deal with the first two. Positions of the legs and the arms and body orientations are what postures mostly composed of (Wainwright, 2011, p. 94). Knowing others' postures as they are standing/sitting allows us to determine 
the most efficient approach to deal with them, Albert Mehrabian shows how postures reflect our mental attitudes:

\subsection{Open or Close}

People with arms folded, legs crossed, and bodies turned away are signaling rejection of the messages being conveyed. On the other hand, people showing open hands, fully facing the audience and (if sitting) both feet are on the ground, they are more open to others, and accepting what the others are saying, they are more willing to interact (positive attitude).

\subsection{Forward or Back}

When people are leaning forward and pointing towards you, even with a small angle, they are actively accepting or criticizing (evaluating) the information given to them. If they are leaning back, looking at the ceiling or probably busy with anything (looking at their fingers, cleaning their glasses, looking around...) they are not interested in the lecture or ignoring it, or they passively absorb the messages being broadcasted. The figure below illustrates the postures of sitting:

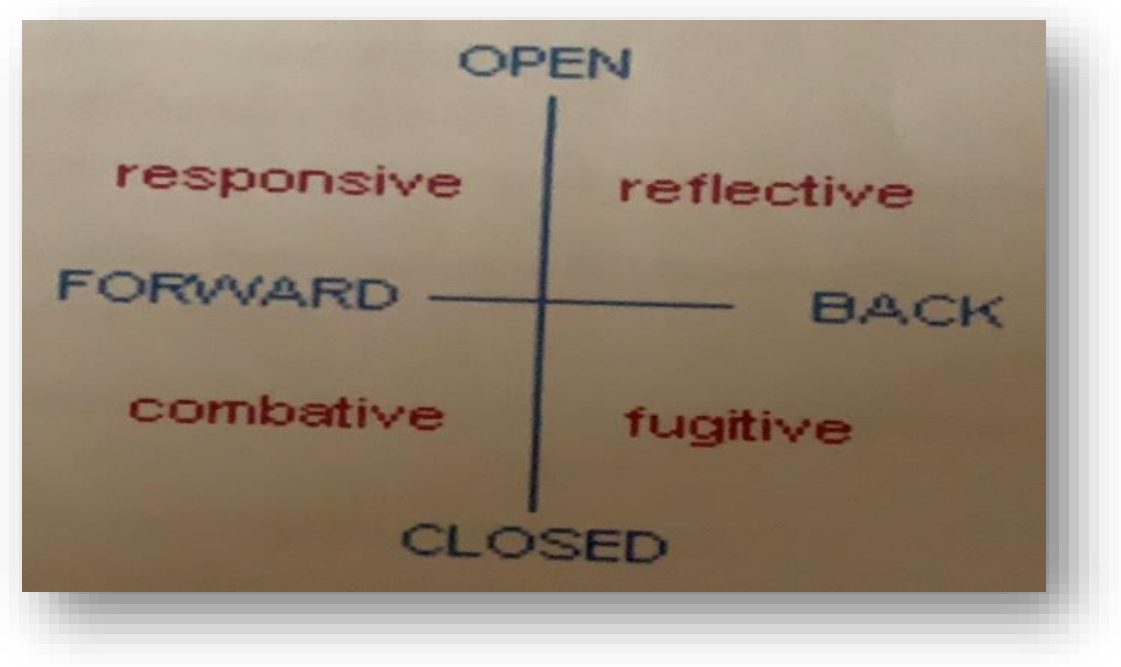

Figure 1: Body orientations, from "Mind your Manners" John Mole.

The figure reveals those attitudes:

1. Responsive: engaged, open postures, ready to agree, attentive.

2. Reflective: attentive, evaluating posture.

3. Fugitive: bored, doodling, rejection, closed posture.

4. Combative: "let me speak" position, sometimes aggressive, defiant (standing).

\subsubsection{Gestures}

Gestures express attitudes, emotions, and non-verbal reactions. They permit a degree of expressiveness that other aspects of nonverbal communication don't. Gestures are important not only in body language 
but also in sign language (the language used by deaf and mute people). According to Gerard Nierenberg and Henry Calero, gestures express attitudes and emotions like openness, frustration, confidence, nervousness...etc. Several scientists classified gestures in categories. For instance, Michael Argyle suggested the following classification of gestures:

- Illustrations and other speech-linked signals.

- Conventional signs and sign language.

- Movements that express emotions.

- Movements that express personality.

- Movements that are related to religious practices and cultural rituals.

Other categorizations done by the psychologist Ekman Friesen and Hager show that gestures are divided according to:

Emblems: movements that substitute words. (Can easily be translated. E.g. the ok sign).

Illustrators: movements that accompany speech.

Regulators: movements signaling the change in listening or speaking roles. (E.g. nodding is a sign to encourage the speaker that you are following.)

Adaptors: movements betraying the person's emotional state, unconscious cues. (E.g. hair twisting, swinging legs).

Affect displays: movements that directly reveal emotions. More related to facial expressions.

Some gestures are universal i.e. they have the same meaning wherever we go. For example: rubbing palms together to show anticipation; yawning as a sign of boredom; shoulder shrug (it can mean: 'I don't know' or 'I don't care' or 'what else can I do?')

The shoulder shrug, however, is a mixture of many gestures: shoulders go rise and fall may accompany crossing arms of open palms. According to the context and the emotional state of the person, also according to how we can read the gestures cluster as will be shown later in this chapter. Some gestures are not universal but cultural; each culture may interpret a gesture as the opposite meaning of another culture.

\subsubsection{Head Tilt}

"Use your head" doesn't mean only think. Head movements are not only important in speaking but also in listening. They can be conscious or unconscious indicating attitudes and preferences they can also replace speech and provide support for what is being said.

Head movements have a major role especially in public speaking, as a speaker or as a hearer. As a speaker, you use your head to support or emphasize your speech, as a hearer, your nodding is a sign that you are interested; if not, your head will loose and will need your hand to prevent it from falling as a sign of boredom and dislike. We can recognize our audience for being active listeners by looking at their heads, if the head is slightly tilted when they are listening to a certain speech, they are actively listening. You can watch children tilting their heads when someone is speaking to them, and even animals (especially dogs) 
if they hear anything that delights them. Nodding can mean many things: agreement, approval, acceptance, attention, and understanding.

\section{Facial Expressions and Eye Contact}

\subsection{Facial Expressions}

A facial expression is a micro-expression that is shown in the face of humans according to the emotions that are experienced. Unlike prolonged facial expressions, micro-expressions are difficult to fake because they occur as fast as $1 / 15$ to $1 / 25$ of a second. Facial expressions are innate and universal; in fact, animals do too have some common facial expressions as human beings have according to studies shown in Charles Darwin's book The Expression of the Emotions in Man and Animals (1872).

Here are the seven universal facial expressions according to the body language expert Vanessa Van Edwards:

a. Surprise: Eyebrows are raised and curved. The skin below the brows is stretched. Horizontal wrinkles across the forehead are manifested. Eyelids are wide open. Jaw drops open and teeth are parted, but there is no tension or stretching of the mouth.

b. Fear: Eyebrows are raised and down together, usually in a flat line. Wrinkles in the forehead are in the center between the brows, not across (vertically). The upper eyelids are raised, but the lower ones are tensed and drawn up. The mouth is open (usually); lips are tensed or stretched and drawn back.

c. Disgust: The upper lids are raised. The lower lip is raised. The nose is wrinkled. Cheeks are raised and lines are shown below the lower lids.

d. Anger: The brows are lowered and drawn together. Vertical lines are shown between the brows. Lower lids are tensed. The eyes star hard or bulging. Lips pressed firmly together with corners down. Dilated nostrils. The lower jaw may come out a bit.

e. Happiness: Lips are drawn back and up. Wrinkles are manifested from outside the nose to the outer lip. Cheeks are raised. The lower lid may show wrinkles or be tensed. (There are real smiles as the description shown above; as there are fake smiles which are characterized by the disengagement of eyes' muscles unlike the real ones).

f. Sadness: The inner corners of the eyebrows are drawn in and up. The skin below eyebrows is triangulated with inner corners up. Corners of the lips are drawn down. The jaw comes up and the lower lip pouts out.

g. Contempt: one side of the mouth is raised (Edwards, 2013).

Ekman, Friesen and Hager (1978) divided a system that allows measuring facial muscle contraction involved in facial expression; it is called "The Facial Action Coding System". This system is used to generate and study different human micro-expressions that help in analyzing their emotions and psychology (Ekman, 1995). Experts and people who work in investigations use the system of reading and analyzing facial expressions to detect lying. When we lie our faces reveal us because we cannot fake our faces "words lie, your face doesn't..." Paul Ekman. Our subconscious mind is working along with our 
body, so if we lie our body will betray us and will send some contradictory cues. That's why people who rarely lie are easier to catch (Pease, 2011).

\subsection{Eye Contact}

"Eyes are the windows of the soul" simply because they reveal so much about our inner self (men are not like women, extroverts are not like introverts and so on).

Eye contact differs according to the context of the situation we are in. Feedback is important when we are speaking because it helps to maintain the interrelation of the communication. We can know also whether people like us or not or whether they are interested and paying attention to what is being said. For example, people who tend to avoid eye contact are feeling embarrassed or shame, or may feel sorrow, yet, we should relate our interpretation with other gestures and behaviors because human beings are unpredictable in their behaviors.

Accordingly, many experiments show that pupils of the eyes tend to dilate if one is interested of what he/she hears, and people with dilated pupils are more likely to be socialized with others. The operation where our pupils dilate is unconsciously done because it is considered to be a response to different stimuli. The use of eye contact is seeking information; displaying attention and interest; inviting and controlling interaction; seeking to influence, dominate or threaten others; providing feedback during the speech; revealing attitudes. The rules that govern the use of eye contact can be linked to the grammar of spoken language because we punctuate our speech with eye contact. Too much eye contact can be considered disrespectful and threatening sometimes. While too little eye contact signifies shyness, lack of interest, lack of attention, dishonesty, and insecurity. According to NLP (Neurolinguistics Programming) researchers: when we process the information our eyes move according to certain patterns:

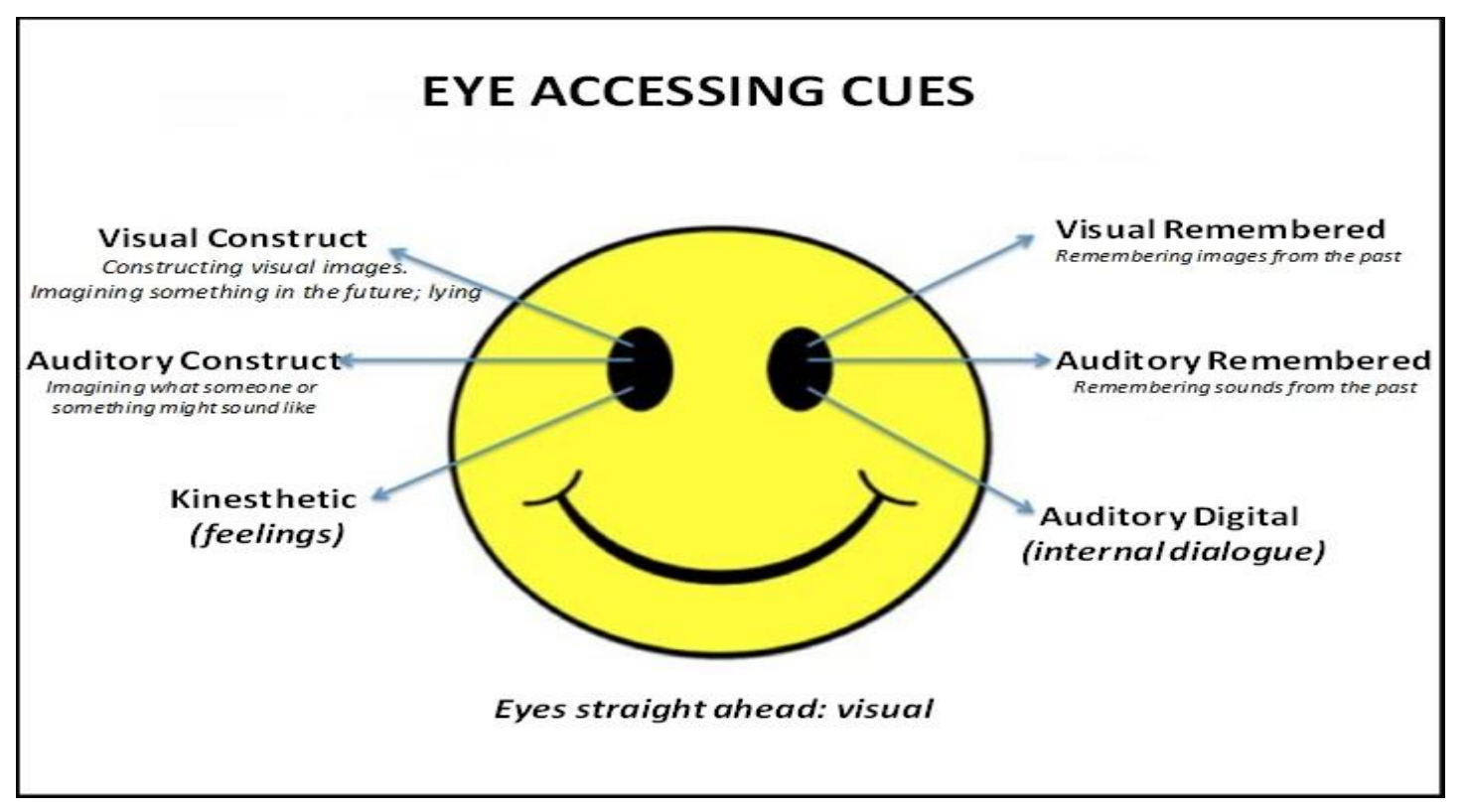

Figure 2: What eye movements mean (from a video on nonverbal communication)

1. Visual thinkers: often use words like 'I see that...', 'that's quite clear to me...' this Throw lights on...' 
2. Auditory thinkers: use words that describe sounds: 'it sounds to me like...', 'listen...'

3. Kinesthetic thinkers: use more emotional language like: 'it feels good to me...'; 'it's getting hard to handle...'

\subsection{Spacing and Timing}

\subsubsection{Spacing}

According to Environmental Psychologist Robert Sommer (1929), personal space is: "...that area around each of us which we do not like others to enter except by invitation or under certain special circumstances". A territory is an area around a person that he/she claims as his/her own, for example, home is bounded by fences to claim personal possession of the zone. The American anthropologist Edward Hall was one of the pioneers in the study of man's spatial needs, and in early 1960's he coined the term 'Proxemics' for the science of distances around human beings, and how it can create relationships. The following figure shows approximately how distances are described in terms of "comfort zones" for the person and different people around him/her:

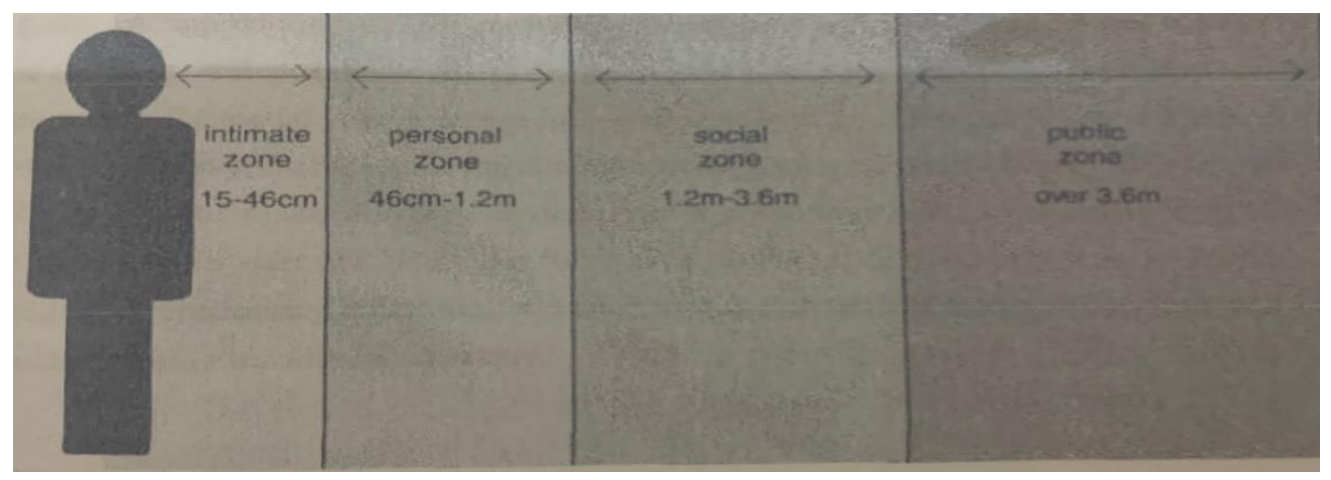

Figure 3. Personal zone Distances

1. The intimate zone: the zone where only close people can invade.

2. The personal zone: an arm length distance or more, usually for friends and extended family members and so forth.

3. The social zone: used in everyday encounters of a social or business nature.

4. The public zone: outwards and strangers.

\subsubsection{Timing}

Time and timing play a major role in nonverbal communication. The long pause, the quick-fire reply, the delayed entrance, the talking and moving speech situation - all reflect the way we communicate. The science known as Chronemics, study the use of time in our interactions. Improving and understanding how we can use the time for our advantage, not only will help us communicate better, but also help us manage our busy lives better. The amount of time we spend communicating with each other is very little, but once it is used effectively it is enough. In that sense, people are different in using their times; some people do best in the morning, whereas some do better at late at night. Scientists have found that the time patterns followed by people are biological according to the circadian rhythms of the body. 
Speakers should know how to choose pauses and silences during their speech, a silence may indicate that the speaker is gathering his/her thoughts, or it may indicate nervousness. However, the absence of pauses in speech may signify that the speaker is rushing and lacking confidence. Silence also can mean: it is time for applause or quick laughter from the audience, and even to emphasize the participation of the other side of the communication. To signal your presence in or during the speech you are required to maintain a sense of timing to successfully gain the attention without interrupting or being rude. If we want to make the best of our time in communication, we need to draw a scheme of when we start and when we finish. We need to make our performance faster and avoid any unnecessary repetition, we need to pick the best moments for moving into new situations using transitions smoothly.

\section{Signals and Nonverbal Aspects of Speech (paralinguistic cues)}

We assume that information is conveyed verbally. Surprisingly, the sounds of our speech and the delivery are just as important as the content of the words themselves. Albert Mehrabian divided communication in face-to-face encounters into three parts: $7 \%$ words, 55\% body language, and 38\% from the tone, inflection, and speed of the voice. The nonverbal aspects of speech form the basis of the study of paralinguistic and it includes:

1. Volume: to be trustworthy you have to choose the right volume of your voice, so it is not too high or too low.

2. The tone of the voice: it should not be too harsh or too smooth.

3. Pitch: changing the pitch while speaking can convey shades of meaning such as emphasis, or surprise, or distinguish a statement from a question (Boundless, 2014).

4. Voice quality: be the clearest possible.

5. Rate of speech: controlling one rhythm, not too slow or too fast.

6. Stress: knowing how and when to emphasize words or phrases without overdoing it. All of these factors are significant to communication because they add emphasis and convey emotions; in addition to that, they provide speech punctuation and pauses, and help the speaker control the direction of his/her speech without indicating nervousness or deception (Wainwright, 2010).

\section{Cultural Differences in Body Language}

The world nowadays is getting smaller, and communication between people all around the world is a must because what is going on in the world concerns everybody. Yet, we sometimes find difficulties in communicating with other people from different cultures though we may know well their language.

People of western countries tend to focus more on body language and eye contact during face-to-face communication while, for instance, Arabs and Muslims find it disrespectful to maintain a long duration eye contact. The Japanese look at other people very little and tend to focus on the face and the neck while conversing. Gestures are also different in many places in the world. One gesture can mean something in the United States, and mean something offensive in another country. Hands and fingers are also different in use; Middle Eastern cultures prevent eating with left hands because they are reserved for bodily hygiene. Some Venezuelans may use their lips to point at things because pointing with a finger is impolite. Though we may differ in many gestures and bodily expressions, we are alike in many others, like universal facial expressions that do not change because they characterize all human beings. The differences may be due to 
social customs or some religious factors in society. We have to take into consideration those differences to show respect and appreciate other cultures because people are more comfortable with those who show good manners and sincerity.

\section{How to Read Body Language?}

To read body language is to understand people's emotional conditions and cultural backgrounds. To read body language accurately, body movements should agree with what is being said (in face-to-face communication). Body language can only be read in contexts and clusters; many gestures occur at the same time so they can't be read separately. Like spoken language, body language has its words, sentences, and punctuation. Each gesture is like a word, and every word has various meanings, only when a word is within a sentence it seems understandable for the reader, exactly like gestures: Word à Gesture Sentence à Gesture clusters. Reading body language makes us good observers, and more aware of people's mentalities and personalities, and therefore providing a suitable environment for mutual understanding in communication. Body language reading also makes us more sensitive to people's emotions and feelings. It helps in improving our relationships with others (Pease \& Pease, 2006).

\section{Men vs Women}

Performing or reading body language can differ between men and women in terms of biological, psychological, and also social factors. Women are different in using their brains in a communicative situation than men. Women tend to be more perceptive because they think more in a descriptive way. Women use their right brain hemisphere more while men use the left one. Women can do four activities at one time; they can talk in several unrelated topics and use five vocal tones to change the subject or emphasize points. Controversially, men when speaking to women they lose the plot after a while, they can only recognize three vocal tones that the women use. When lying, women tend to make themselves look busy while they are lying, but men usually cover their mouths or touch their noses while telling a lie. Overall, it is easy for body language experts to detect liars no matter how good they are at lying. Faking body language is possible but not for a long time because when you long predict something that does not represent you, your body will reveal it by showing contradictory actions. Just like President Bill Clinton and the Scandal of Lewinsky, experts knew he was lying by interpreting his unconscious body movements that displayed lying.

\section{Do's and Don'ts for Teachers on Body Language}

Why do teachers need to pay attention to their body language? Simply, because it is what makes students respond to the teacher and their output. Researchers have shown that Students do not respond to the content of the lesson if the way of presenting it is poor. Here are some basic instructions for teachers about how to use body language effectively:

\section{a) Use your Voice}

Teachers perform their lessons as they perform to sell an idea to their audience, with their voice, gestures, and facial expressions. As a teacher, try to address your class with clear and upbeat voice, that's how you take control of your students' divided attention. Nothing is more frustrating for students than struggling to hear the low voice of your lecturer (or those who mumble, stutter...) inside the classroom (Grenard, 2012). 


\section{b) Avoid Standing Behind the Table for Too Long}

Standing behind the table for a long time may create a physical barrier between the teacher and his/her students, and may establish gaps in communication during class, especially teachers who tend to sit all the session on their chairs with no motion or lack of it. Those teachers were described by the majority of students attending as "boring".

\section{c) Use Your Space}

Occupy space in a way that shows you are comfortable in your class. The way you stand and move on stage demonstrate confidence, and will lead your audience to trust you and what you are presenting to them. Over moving, on the other hand, may confuse and distract your students. Try to find a way to use space effectively.

\section{d) The Power of Face}

The face is very important in any communication. Facial expressions are very helpful in establishing authentic teaching/learning experience, in which students will depend mostly on the face of the teacher to enhance the deliverance of the lesson. The more expressive your face is, the more students will pay attention and engage more in participating to achieve the aim of the class.

\section{e) Hands and Gestures}

According to Vanessa Van Edwards2, our hands play a major role in indicating trust. It is said that back to cavemen life when people encounter, the first thing to do was checking the others' hands for weapons or physical threats so that they can or cannot trust each other. Behaviorists say that this is a survival trick that we still use unconsciously to detect trust-worthy people. Therefore, teachers need to keep their hands open and use them relevantly with verbal communication to indicate trustworthy to their students and gain their attention (Edwards, 2014).

\section{f) Standing Tall}

This will inspire confidence and credibility and will show how charismatic the teacher is. It will also tell students that the teacher is maintaining control in the classroom.

\section{g) Moving/ Talking Slower}

Rushing in motions may indicate nervousness. Students are always looking for their teachers for how they feel and look because they will respond similarly. Correspondingly, slowing down the rate of your speech will calm the atmosphere, and help the students better understand and memories what is being explained. Teachers have to be flexible in dealing with their students, and with the subject matter being discussed, using body language as a tool to aid accomplishing the job.

\section{Getting the Student's Attention}

In a lesson, your first task is to draw your student's attention to the material that you are trying to present. Attention-getting skills are important in keeping control and avoiding waste of time in the beginning, and 
during the lesson. Use markers, which are signals to hook the class's attention that the lesson will begin, or it will continue. Markers convey no information, but they reassert the teacher's control over the classroom and save time by ensuring messages not to be repeated. Some of these markers could be banging a board rubber or rolling pin on a table and so on. These kinds of markers hold a limited significance, for example, telling the students to be quiet. They also give the floor to the teacher to start his/her lecture in a confident way. Because when you call students to pay attention and you seem to be uncertain, that will create a potential problem if you don't know how to manage public speaking. Having the ability to maintain an eye contact that is well distributed among students will assure your leadership as a teacher and have what Morris interpreted as: "a class falls in love with their teacher at first sight!" A teacher has to show students that she/he is certain. Avoiding the class gaze by looking at the teacher's notebook or register, for instance, will show students that their teacher is uncertain and nervous in some instances. Looking uncertain will affect the teacher's credibility, and will result in poor communication during class. Also, when the teacher avoids looking at someone's eyes while talking to them, it is interpreted as a lack of interest in them, and it is not a good start for gaining the class's respect and approval (Neill, Casewell 1993).

A teacher should try to look confident as much as possible by behaving casually, but in an appropriate way; looking confident means being comfortable but without using loose postures. Many loose postures are used by teachers, amongst them we may be more familiar with "standing with hands in the pockets and leaning or sitting on furniture". It may make the teacher feels comfortable, but it will affect the student's attention and may show that the teacher is not displaying mutual respect. If the teacher is nervous though, there are some techniques to hide his/her nervousness without hinting anything to students. Teachers should know that teaching involves constant stress, and only experienced teachers can know stress patterns and control them; unlike inexperienced teachers, those patterns may be a big deal to handle, especially when the teacher tries to catch the class's attention at first encounters. How to get the class's attention should not be a major section of the lesson in terms of time. Having got a student's attention, the teacher should rapidly move to achieve the aim of his/her lesson, using some non-verbal techniques that were shown above. If you are to maintain that attention for the rest of the lesson, let alone for a whole year, you must communicate to them that what you are about to teach is interesting and worth learning, because initially, body language is just a helping tool to get to what is more important: having a command over the content of the lesson.

\section{Effective Teaching}

Effective teaching occurs when the teacher knows how to convey enthusiasm. Enthusiastic teachers use a wide range of facial expressions, intonation, and gestures to convey the interest end excitement of the subject matter. You can express interest by many signals and supportive responses to errors, at least at the beginning of the sessions. Below are some graphic descriptions on how effective teachers do during their lessons, taking from the video study of Neill 1991 (Neill \& Casewell 1993, p. 90-105), with a brief explanation of each one: 


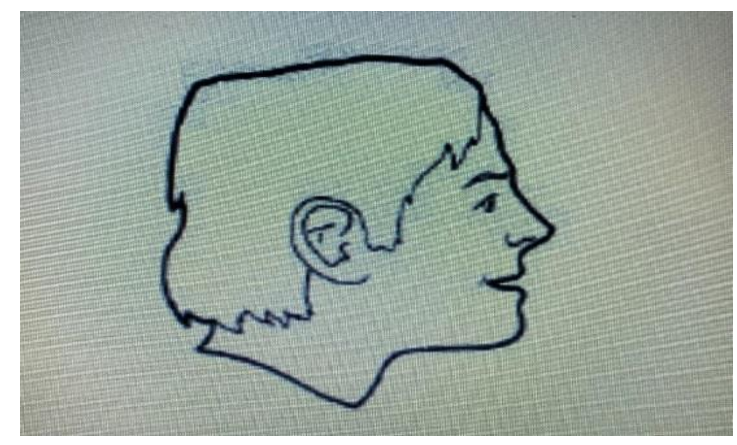

Figure 4

The head of the teacher is forward, with a smile on his face, a relatively mild expression of interest in what the student has to say. Most students saw this teacher as "friendly", and gave a mixture of other positive comments.

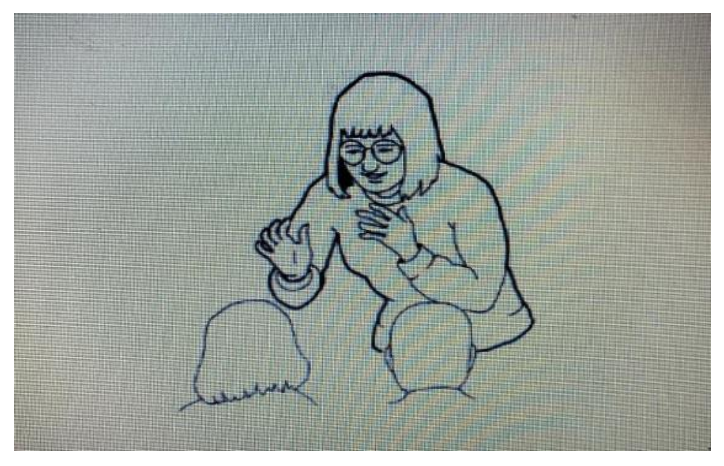

Figure 5

The teacher here is effectively catching the idea of the student, with a lean that most students (especially children) see it as helpful, though some may find this approach threatening and overpowering.

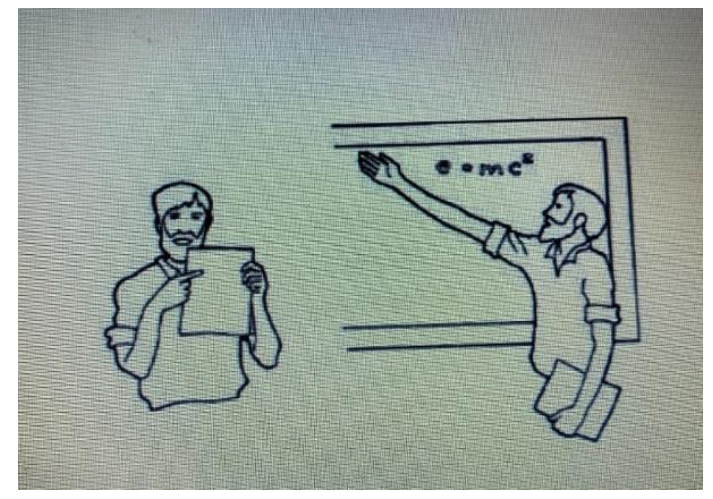

Figure 6

Using gestures to indicate "see what I mean" to point at many things, providing students to follow you. For example: pointing by fingers (the left), and using your hands in indicating things on the board (at the right). 


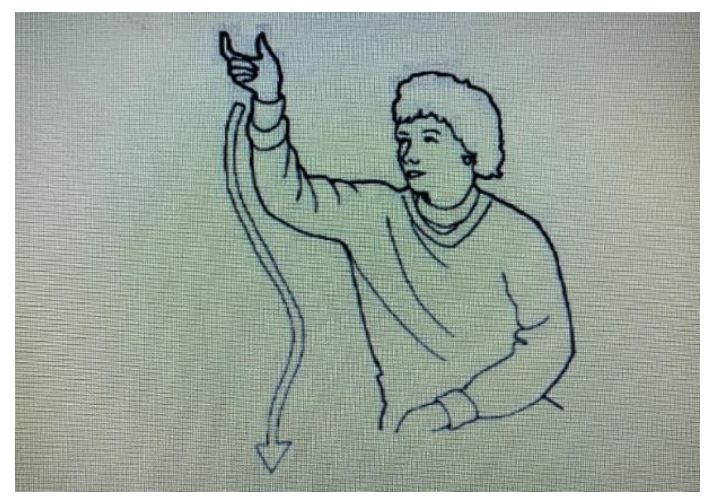

Figure 7

Making abstract notions concrete by gestures and motions. This teacher, for instance, is miming the course of World War 1 trenches down between Belgium and Switzerland.

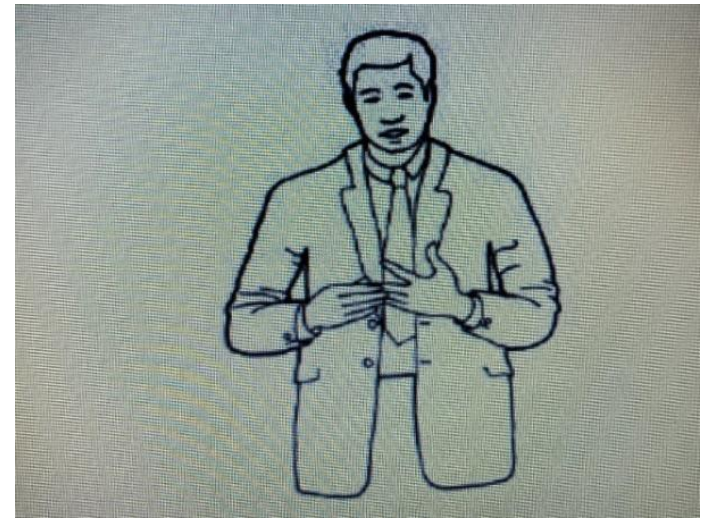

Figure 8

Counting points that are made verbally is a helpful way to make students draw an image about what is being said, and help them remember each element separately. Those were some situations effective teachers use to convey their message enthusiastically. Yet, those gestures and postures are not limited; each teacher is going to decide which one to make to get his/her goals achieved using different body language tools that have been mentioned all along with the two chapters of this memoir (Neill Casewell, 1993).

\section{Sitting Arrangement}

Certain students choose to sit in specific positions inside the classroom; each position can affect the outcome of the lesson. Where a person sits concerning other people consists to be an effective way of obtaining co-operation from them. Dr. John Kershner of Ontario Institute for Studies in Education studied teachers and recorded where they were looking every 30 seconds for 15 minutes. He found that teachers almost ignored students on their right. The study showed also that teachers looked straight ahead by a percentage of $44 \%$, to the left by $39 \%$ and to the right by only $17 \%$ (Pease \& Pease, 2004, p339-40). According to the results of the study conducted by Kershner, students who sat on the left performed better 
in spelling tests than those who sat in the right. Also, people who sit in the front row learn and retain more than others in the audience, partially because those in front are keener than others to learn, and they show more attention to the speaker, simply because they are closer. Those in the middle are the next more attentive and ask the most questions. The side areas and the back are the least responsive and attentive. When the teacher stands on the right side of the stage (the audience's left side), his/her information will have a stronger effect on the right brain hemisphere of the audience's brains, which is the emotional side in most people. Standing to the audience's right (the left side of the stage) is going to affect their left brain hemisphere and will not create the response that the teacher wants. Robert Sommer found that there is a "learning zone" in the classroom shaped like a funnel.

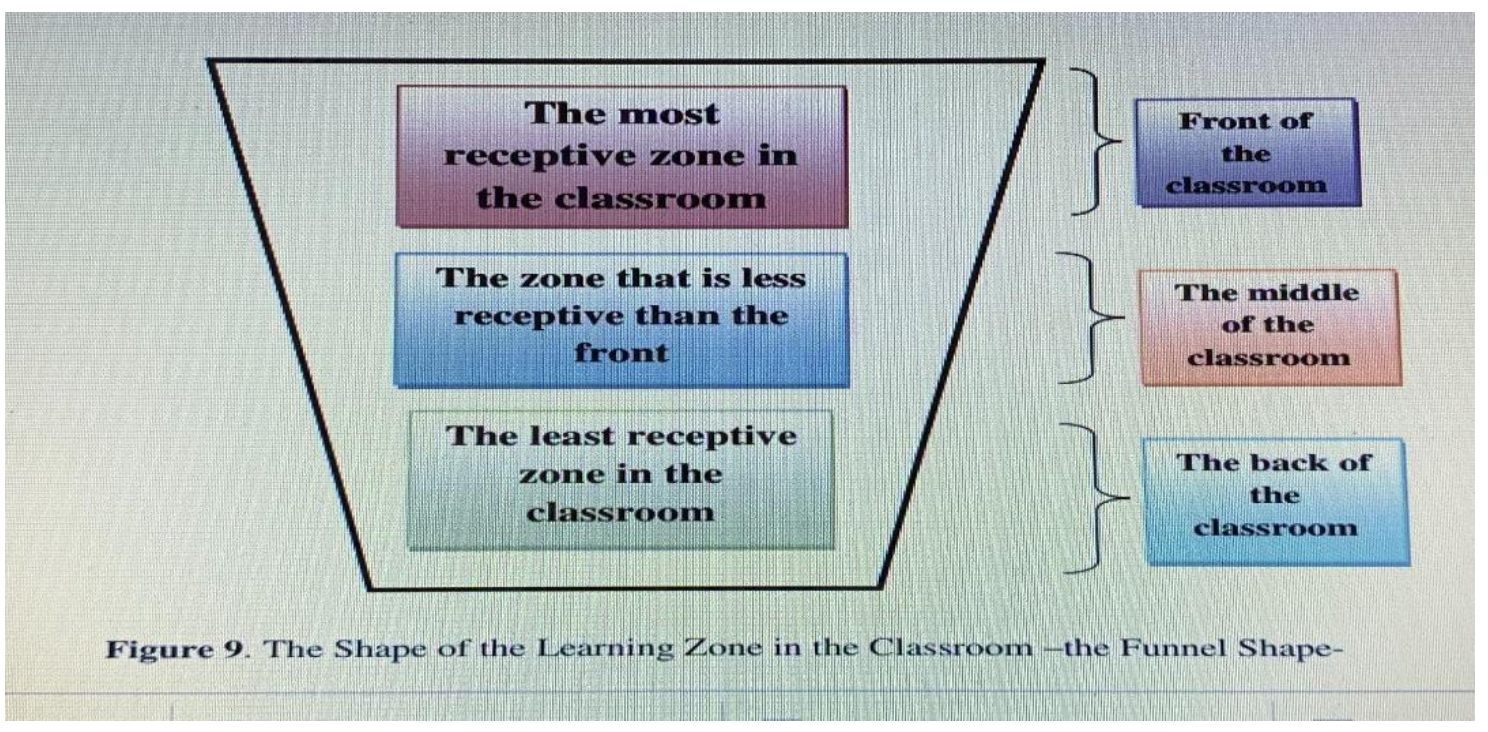

An experiment was conducted to determine whether the funnel effect as a result of where students chose to sit, based on their interest in the topic, or whether the seat the person sat in affected their participation and retention. The experiment is placing name cards on delegates' seats so they could not take their usual positions, enthusiastic people were intentionally made to sit in the sides and the back, and well-known back-row people in front. It has been found that this strategy did not only increase the participation and recall of the negative delegates who sat in front, but also decrease the participation and recall of the usually positive delegates who had been relegated to the back. Basically, people who sit in the area which draws directly down the center of an audience and across the front row (the funnel shape/ the learning zone) participate the most, and have the highest recall; whereas people who participated the least and had the lowest recall are those who sat in the back or the sides. The analysis of this study highlights a clear teaching issue. In this respect, some teachers and trainers have abandoned the traditional "classroom style" sitting arrangement and replaced it by the "horseshoe" or the "open square" arrangement, because evidence suggests that those kinds of arrangement produce more participants and better recall of each individual (Pease \& Pease, 2004).

\section{The Student Body Language}

Students inside classrooms show nonverbal behaviors when responding to their teacher's inputs. These behaviors help the teacher to know how to manage his/her class effectively to gain students' attention. 


\subsection{Boredom}

During the lesson, when your students begin to use gestures like using their hands to support their heads, it indicates that they are feeling bored. The student usually starts with his/her chin being supported by the hand or thumb, but the whole cluster of hand supporting the head from falling asleep is the most popular sign of boredom, besides yawning, putting fingers in one's hair, gossiping with others, or even putting the head on the table.

Many other gestures like drumming fingers on the table, looking around, or making themselves busy with something else, are all indicators of the lack of interest. After the teacher notices that, he/she has to change the direction of the talk so that students renew their interest.

\subsection{Active Students}

Mostly, active students will appear in the class more than the others. Nodding and head tilting, for instance, are good signs that indicate attentiveness, and that the student is interested in what is being said. Different facial expressions are identified by the teacher, which indicate the student's listening. Eye contact when maintained between the teacher and the students will help to establish communication inside the classroom, and therefore actively contributing students in the lesson.

\section{Conclusion}

Humans communicate by other means besides spoken language. We tend to accompany our words with other behaviors because only information is conveyed through words, the emotional and attitudinal information is conveyed through other means like gestures, eye looks, tone of voice.....and so forth. Body language has several types according to the body part used, and also according to one's psyche. Some of our gestures and body movements are universal, yet many of them are cultural or religious. Knowing the difference in gestures across cultures helps expressing respect and appreciation to that culture, and also facilitates the process of communication with people from it. Learning how to read general body language can help in improving ourselves and increase one's self-esteem. Furthermore, reading others' body language helps us better control our relationships, because mainly we will know the psychological and the emotional state, besides the culture of the person we are trying to communicate with. How body language can be a powerful tool to help the teacher managing a successful class and establish an authentic relationship with students was the main core of this research. Also, how can teachers get their students' attention, and detect bored students by turning them into active contributors to the lesson have been dealt with in this memoir? This research emphasizes the role of nonverbal communication that comes along with verbal communication in establishing and maintaining contact or interaction between people and the environment. In teaching, slow-learners appreciate those teachers who make the learning situation conducive, and fruitful through their effective teaching process with the help of face and relevant body language. Moreover, if deaf and mute people can learn with gestures and facial expressions, I concluded that using body language alone can be sufficient to transfer a message.

Our bodies are the mirrors of our inner self, that's why it is important to be aware of how to work with it, and how can you make it an advantageous tool to increase your influence as a teacher, a public speaker, a student, or even as an ordinary person. Although many works and investigations have been done on 
nonverbal communication in general, and body language in particular; some many sociologists and psychologists are not convinced with the fact that nonverbal communication is a vital part in face-to-face communication. Specifically, criticizing what Mehrabian explored as the "7\% Rule" which states that the actual words uttered in face-to-face communication accounted for only $7 \%$ and the rest of the information is divided between vocal variety and body movements. Is it really accurate to say that words account for only $7 \%$ of the whole communication process? Why Mehrabian excluded the importance of words and emphasized on nonverbal communication? It is still a debatable issue among behaviorists and psychologists on which is more important what you say, or HOW you say it.

\section{References}

Chisholm, H. (1911). Johann Kaspar Lavater. Encyclopædia Britannica. Cambridge University Press.

Darwin, C. (1965). The expression of the emotions in man and animals. Chicago: University of Chicago Press.

Ekman, P., Wallace V. F., \& Hager, J. (1978). The facial action coding system (FACS): A technique for the measurement of facial action. Palo Alto. CA: Consulting Psychologists Press.

Genard, G. (2012). 5 Key Body Language Tips of Public Speaking. The Genard Method. Retrieved from http://www.genardmethod.com/blog-detail/view/135/5- key-body-language-tips-of-publicspeaking

Highfield, R., Wiseman, R., \& Jenkins, R. (2009). How your looks betray your personality - life - New Scientist. Newscientist.com. Retrieved 25 May 2014, from http://www.newscientist.com/article/mg20126957.300-how-your-looks-betrayyourpersonality.html

Key, M. (1970). La linguistique. Presses Universities De France.

Key, M. (1980). The relationship of verbal and nonverbal communication. The Hague: Mouton.

Neill, S., \& Caswell, C. (1993). Body language for competent teachers. London: Routledge.

Oestreich, H. (1999). Let's Dump the 55\%, 38\%, 7\% Rule. Transitions, 7(2), 11-14.

Pease, A., \& Pease, B. (2004). The definitive book of body language. Buderim.

Phernology. (2015). Retrieved from http://dictionary.reference.com/browse/phrenology Physiognomy.

Merriam-Webster. Retrieved from http://www.merriamwebster.com/dictionary/physiognomy Encyclopedias:

Sharma, V. (2011). Body language: The art of reading gestures \& postures. New Delhi: V \& S Publishers.

Van Edwards, V. (2013). Guide to Reading Micro-expressions - Science of People. Science of People.

Retrieved 29 November 2014, from http://www.scienceofpeople.com/2013/09/guide-readingmicroexpressions/

Van Edwards, V. (2014). 5 Body Language Tips for Teachers - Science of People. Science of People.

Retrieved 4 February 2015, from http://www.scienceofpeople.com/2014/05/5-body-languagetips-teachers/

Waller, B., \& Micheletta, J. (2013). Facial expression in nonhuman animals. Sage Journals. Retrieved 6

December 2014, from http://emr.sagepub.com/content/5/1/54.short Pitch.

Wainwright, G., \& Thompson, R. (2010). Understand body language. London: TeachYourself. 